\title{
Editorial: \\ Introduction to the Special Issue on Adaptive and Reconfigurable Distributed Systems
}

\section{Background}

An adaptive and reconfigurable software distributed system can repair itself if any execution problems occur. This adaptation aims to successfully complete its own execution by respecting functional and non-functional agreements. The system should be able to predict or to detect degradations and failures as soon as possible to analyze the situation, and enact suitable recovery actions.

Several aspects have to be considered in the design and the implementation of adaptive and reconfigurable distributed systems, for instance, the monitoring, diagnosis, decision and runtime execution of distributed systems. These aspects are applied in different investigation topics such as component-based software engineering, service oriented architecture, cloud applications, business process, and nonfunctional requirements (like QoS, performance, resilience).

This special issue addresses topics related to the aspects presented above. We received twenty-three papers dealing with these topics. Six articles were selected, which means an acceptance rate of $26 \%$. Each submitted article was evaluated by at least two reviewers during two or more rounds of review for accepted ones. The accepted articles are devoted to recent research addressing both theoretical and practical aspects on adaptive and reconfigurable distributed systems. An overview of the proposed contributions is presented in the sequel.

\section{Accepted papers in this special issue}

In the first paper, Alkafaf et al. [1] use the local and global knowledge base for developing self-adaptive systems. The local knowledge base is used for making an initial decision to identify an encountered object. The global knowledge base is used when the object cannot be identified. The knowledge base is exposed as a web service that is shared by multiple self-adaptive units for collaborative decisions. The authors implemented the proposed hybrid approach using Robot Operating System, a software framework providing operating system-like functionality for developing robotic software. They also evaluate the proposed approach under a 3D simulation environment for robotic systems.

In the second paper, Gassara et al. [2] present a formal approach for designing correct deployment architectures using the formal technique of Bigraphs and Bigraphical Reactive Systems. The proposed approach uses an architectural refinement process based on multi-scale modeling, which allows the analysis of the architecture through different abstraction levels. The passage from a scale to another is also done in a formal way, through the application of various rules of architectural transformation. To facilitate the understanding of the proposal, a case study called smart home is described. It illustrates, in a simple way, the application of the approach in its different scales including aspects of architectural reconfigurations

Souza et al. [3] propose an approach for monitoring dynamic service-oriented applications. They propose a meta-model that represents service, quality, and event domains, which represents the abstract syntax of a domain specific language. Based on the defined meta-model, the designer can define provided and required services, the quality attributes and metrics. He can also specify how these metrics can be defined and computed based on a set of events thrown while the application is running. As proof of concepts, the authors designed and implemented a dynamic service oriented architecture platform.

Rekik et al. [4] present a comprehensive end-to-end framework for business process outsourcing to the cloud, which considers the dynamic business process context. The proposed framework comprises different methods able to efficiently take into account accurate and up-to-date business process context to identify the best process fragment to outsource and the most suitable cloud service to adopt.

The optimality of the identified solutions is insured by NSGA (Elitist Non-Dominated Sorting Genetic Algorithm) for which the authors proposed a set of context-based objective functions. In addition, the 
framework considers the business process context prior and posts the outsourcing decision in order to change a decision to account for context changes.

Hacid et al. [5] propose an approach for selecting executors of business processes tasks based on two criteria: profit and social-qualities exhibited by executors (e.g., selfishness). Based on a trusted authority, the proposed approach ensures the transparency and fairness of this selection. The approach also offers business processes owners the means to adapt their selection strategies based on these criteria. The authors present an implementation of the proposed approach and evaluate it using a set of simulations.

Alférez and Pelechano [6] propose to use models at runtime to guide autonomic adjustments of contextaware service compositions. For each problematic event, the system itself can query models during execution to determine the necessary modifications in the service composition. These modifications correspond to adding or removing fragments of WS-BPEL code that can be deployed at runtime. In addition, the authors present an implementation and a promising evaluation of the proposed framework.

We would like to express our sincere thanks to the editorial staff for their support and help. We also thank all the authors who submitted their papers, and the thoughtful work of the many reviewers who have provided rich evaluations and recommendations in a timely manner.

\section{References}

[1] Dhrgam AL Kafaf and Dae-Kyoo Kim, A Web Service-Based Approach for Developing SelfAdaptive Systems, Computers \& Electrical Engineering

[2] Amal Gassara and Ismael Bouassida Rodriguez, Describing Correct Deployment Architectures Based on a Bigraphical Multi-scale Modeling Approach, Computers \& Electrical Engineering

[3] Fabio Souza, Tarcisio Coutinho, and Nelson Rosa, Monitoring Solution in a Dynamic ServiceOriented Platform, Computers \& Electrical Engineering

[4] Mouna Rekik, Khouloud Boukadi, and Hanene Ben-Abdallah, An end-to-end framework for contextaware business process outsourcing to the cloud, Computers \& Electrical Engineering

[5] Hakim Hacid, Emir Ugljanin, Mohamed Sellami, and Zakaria Maamar, Adapting Selection Strategies of Executors of Business Processes based on Profit and Social Qualities, Computers \& Electrical Engineering

[6] Germán H. Alférez and Vicente Pelechano, Achieving Autonomic Web Service Compositions with Models at Runtime, Computers \& Electrical Engineering

\section{Guest Editors}

Slim Kallel

ReDCAD Laboratory, University of Sfax, Tunisia

E-mail: slim.kallel@ fsges.rnu.tn

(Managing Guest Editor)

Khalil Drira

LAAS-CNRS, Univ. de Toulouse, France

E-mail: drira@laas.fr

Mohamed Jmaiel

Digital Research Center, Sfax, Tunisia

E-mail: mohamed.jmaiel@ @redcad.tn

\section{Bibliographies}




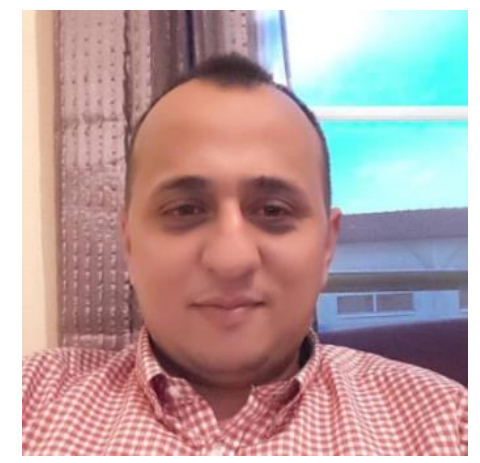

Slim Kallel obtained his diploma of master degree in computer science from National Engineering School of Sfax (Tunisia) in 2005 and his $\mathrm{Ph}$.D. from Darmstadt University of Technology (Germany) in 2011. He joined the University of Sfax (Tunisia) as Assistant Professor of Computer Science at FSEGS in 2009. He became an Associate Professor in 2012. His work focuses on formal methods, business process, and adaptive systems. He is co-chair of the AROSA track (Adaptive and Reconfigurable Systems and Architectures.) at WETICE conference. More details are available on his home page: http://www.redcad.tn/members/kallel/

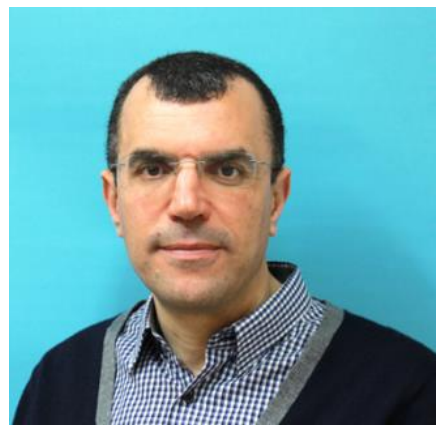

Khalil DRIRA is "Research Director" at LAAS-CNRS, a full-time tenured research position at the French National Center for Scientific Research (CNRS). His research interests include formal design, implementation, testing and provisioning of distributed communicating systems and cooperative networked services. His research activity addressed and addresses different topics in this field focusing on modelbased analysis and design of correctness properties including testability, robustness, adaptability and reconfiguration. Khalil DRIRA is or has been involved in several national and international projects in the field of distributed and concurrent communicating systems. He is author of more than 250 regular and invited papers in international conferences and journals. He is or has been initiator of different national and international projects and collaborations in the field of networked services and distributed and communicating systems. Khalil DRIRA is or has been member and chair of the programme committees of different international and national conferences. He is member of the editorial board of different international journals in the field of software architecture and communicating and distributed systems. Khalil DRIRA has been editor of a number of proceedings, books and journal issues in these fields. More details are available on his home page: http://homepages.laas.fr/khalil

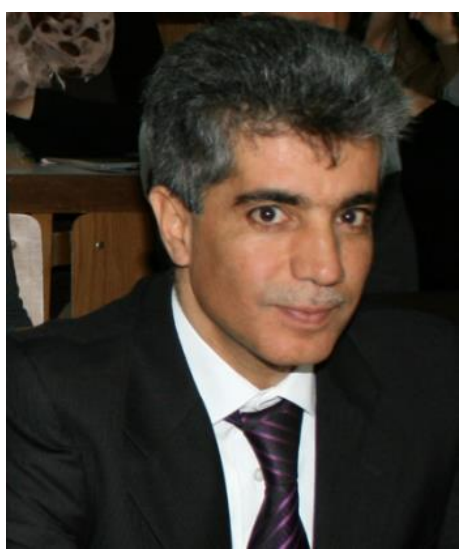

Mohamed JMAIEL obtained his diploma of engineer in Computer Science from Kiel (Germany) University in 1992 and his Ph.D. from the Technical University of Berlin in 1996. He joined the National School of Engineers of Sfax (Tunisia) as Assistant Professor of Computer Science in 1995. He became an Associate Professor in 1997 and full Professor in January 2009. He participated to the initiation of many graduate courses at the University of Sfax. His current research areas include software engineering of distributed systems, formal methods in model-driven architecture, self-adaptive and pervasive systems, autonomic middleware. He conducted many research projects and published more than 150 regular and invited papers in international conferences and journals, and has co-edited four conferences proceedings and three journals special issues on these subjects. He organized and co-chaired the program committees of many international conferences, like MCSEAI'2004, CRiSIS'2009, NOTERE'2010, OPODIS'2010, WETICE'2013, VECOS'2016, and ESBM'2017. He was director of the National Engineering School of Sfax (ENIS), from 2011 to 2014. Currently, he is director of the digital research center at the Technopark of Sfax. More details are available on his home page: http://www.redcad.org/members/jmaiel/ 
